\title{
Are Post Hoc Analyses on Subgroups Sufficient to Support New Treatment Algorithms of Heart Failure? The Case of SGLT2 Inhibitors Associated with Sacubitril/Valsartan
}

\author{
Massimo Volpe $^{\mathrm{a}}$ Giovanna Gallo $^{\mathrm{a}}$ Shelley Zieroth ${ }^{\mathrm{b}}$ \\ ${ }^{a}$ Cardiology Unit, Department of Clinical and Molecular Medicine, Sant'Andrea Hospital, Faculty of Medicine and

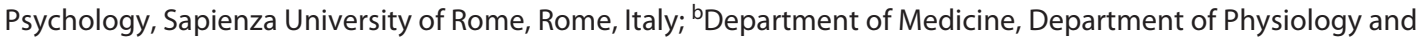 \\ Pathophysiology, St Boniface Hospital, University of Manitoba, Winnipeg, MB, Canada
}

\section{Keywords}

Sacubitril/valsartan · Sodium glucose cotransporter 2 inhibitors · Heart failure

\begin{abstract}
The use of sodium glucose cotransporter 2 inhibitors (SGLT2i) in heart failure (HF) with reduced ejection fraction (HFrEF) has been strongly supported by the results of recent randomized clinical trials. Upon this evidence, international recommendations and consensus documents propose the inclusion of SGLT2i among the first-line classes for HFrEF management. Subsequent analyses of treatment subgroups have been performed to investigate the effects of SGLT2i in patients treated with first-line classes including sacubitril/ valsartan (Sac/Val), showing a consistent reduction of cardiovascular outcomes with a good safety profile of SGLT2i in combination with the other classes. Accordingly, SGLT2i are recommended also in combination with Sac/Val. This association, however, may require caution before being translated into guideline-directed medical therapy in clinical practice, since the proportion of patients receiving Sac/Val and SGLT2i in the available studies was poorly represented. In order to support an effective and safe sequencing or a simultaneous initiation of these 2 drug classes, pragmatic and real-world clinical studies would be helpful.
\end{abstract}

(c) 2021 S. Karger AG, Basel
The development of sodium glucose cotransporter 2 inhibitors (SGLT2i) and their clinical use in heart failure (HF) with reduced ejection fraction (HFrEF) also in diabetic patients have been strongly supported following the publication of 2 successful large randomized controlled trials. The DAPA-HF (Dapagliflozin and Prevention of Adverse Outcomes in Heart Failure) [1] and EMPERORReduced (Empagliflozin Outcome Trial in Patients with Chronic Heart Failure and a Reduced Ejection Fraction) [2] trials, which compared dapagliflozin and empagliflozin, respectively, with placebo on top of standard-ofcare therapy, showed significant reductions of primary endpoint event rates in HFrEF treated with SGLT2i. Upon this evidence, recent international recommendations and consensus documents propose the inclusion of SGLT2i among the first-line classes for HFrEF management [3].

Subsequent analyses of treatment subgroups have been performed to assess whether the benefits of SGLT2 $\mathrm{i}$ on cardiovascular outcomes are uniform and are confirmed when combined with other disease-modifying drugs. In particular, analyses have been attempted to investigate the effects of SGLT2i in patients treated with sacubitril/valsartan (Sac/Val) [4].

In a recent article based on a prespecified subgroup analysis (1 of 12) of the EMPEROR-Reduced [5], Packer and colleagues reported that the favorable effects of em- 
pagliflozin in patients with HFrEF were preserved in the subgroup of patients treated with Sac/Val. For most endpoints (including the primary endpoint of cardiovascular mortality and HF hospitalizations), the magnitude of the effect of empagliflozin in patients receiving Sac/Val tended to be numerically larger than that in those not receiving Sac/Val, although the interaction $p$ values were not statistically significant [1]. However, only 727 subjects were treated with Sac/Val, representing the $19.5 \%$ of the overall study population. Among them, 340 received both empagliflozin and Sac/Val [1]. On the other hand, patients treated with renin-angiotensin-system (RAS) blockers, $\beta$-blockers, and mineralocorticoid receptor antagonists (MRA) were well represented, being 71\%, 95\%, and $70 \%$ of the study population, respectively.

A similar finding was described by Solomon et al. [6] in the subgroup analysis of DAPA-HF. Again, there was consistency of treatment benefit with dapagliflozin in favor of a reduction of the composite primary endpoint in the Sac/ Val subgroup which was not different from what was observed in the non-Sac/Val group. Again, 508 subjects (10.7\% of the entire study population) were treated with Sac/Val in DAPA-HF; of these, only 250 received both dapagliflozin and Sac/Val. A much larger proportion of patients was treated with diuretics (93\%), RAS blockers (85\%), $\beta$-blockers (96\%), and MRA (71\%) [2, 7]. Similarly, in the SOLOISTWHF (Effect of Sotagliflozin on Cardiovascular Events in Patients with Type 2 Diabetes Post Worsening Heart Failure) trial [8], patients receiving background therapy with $\mathrm{Sac} / \mathrm{Val}$ were underrepresented, accounting for only $15 \%$ (92 patients) of the active treatment group.

Based on the subgroup analyses of the EMPEROR-Reduced and DAPA-HF trials, a recently published metaanalysis not surprisingly confirmed the benefits of SGLT2i in patients treated or untreated with Sac/Val [9], a finding confirmed by retrospective studies in patients with $\mathrm{HFrEF}$ and diabetes mellitus [10, 11].

The association of Sac/Val and SGLT2i in HFrEF appears to be safe, since a hypothesized adverse effect of a synergistic blood pressure reduction, mostly linked to increased diuresis, is not supported by subgroup post hoc analyses of EMPEROR-Reduced [5] and DAPA-HF [6].

In light of the abovementioned reports, SGLT2i are currently recommended also in combination with Sac/ $\mathrm{Val}[3,12-14]$. These results $[4-6,8]$, however, may require caution before being translated in clinical practice.

In fact, while the effects of SGLT2i in patients with HFrEF have been demonstrated in randomized clinical trials on top of guideline-directed medical therapy with RAS blockers, $\beta$-blockers, MRA, or loop diuretics $[1,2]$, the proportion of patients receiving Sac/Val was poorly represented, and this may unfortunately undermine definite recommendations.

In addition, studies with Sac/Val did not report the use of SGLT2i [15-18].

Even though Sac/Val and SGLT2i may have a synergistic action on diuresis/natriuresis, inhibition of the sympathetic nervous system, and prevention of adverse cardiac remodeling, with potential additive beneficial effects, there are no preclinical studies investigating this combination.

In order to support an effective and safe sequencing or as some propose simultaneous initiation of the 4 classes as a new standard of care for HFrEF, pragmatic and realworld clinical studies would best evaluate the combination of these 2 drug classes. This virtuous effort would represent, in our view, a key step to further support the paradigm of simultaneous initiation of $\mathrm{Sac} / \mathrm{Val}$ and SGLT2i in the management of HFrEF.

\section{Conflict of Interest Statement}

Massimo Volpe reports personal fees for speaker bureau and/ or consulting in Advisory Board from Amgen, Thousand Oaks, CA, USA; AstraZeneca, Cambridge, UK; Daiichi-Sankyo, Tokyo, Japan; Menarini Int, Florence, Italy; MSD, Kenilworth, NJ, USA; Novartis Pharma, Basel, Switzerland; and Novo Nordisk, Plainsboro, NJ, USA, outside the submitted work. Giovanna Gallo was sub-investigator of the EMPEROR-Reduced and EMPEROR-Preserved trials sponsored by Boehringer Ingelheim, Ingelheim, Germany, and of the PARAGON-HF trial sponsored by Novartis, Basel, Switzerland. Shelley Zieroth reports personal fees for speaker bureau and/or consulting in Advisory Board from Abbott, Lake Bluff, IL, USA; Akcea, Boston, MA, USA; AstraZeneca, Amgen, and Alnylam, Cambridge, MA, USA; Bayer, Leverkusen, Germany; Boehringer Ingelheim, Eli-Lilly, Indianapolis, IN, USA; HLS Therapeutics, Etobicoke, ON, USA; Janssen, Beerse, Belgium; Merck, Darmstadt, Germany; Novartis; Novo Nordisk; Otsuka, Tokyo, Japan; Pfizer, New York, NY, USA; Servier, Suresnes, France; and Vifor, St. Gallen, Switzerland. She also is national lead, steering committee member, or site investigator for clinical trials sponsored by AstraZeneca, Bayer, Boehringer Ingelheim, and Eidos, San Francisco, CA, USA; and Novartis outside the submitted work. She is one of the Journal's editorial board member.

\section{Funding Sources}

The authors have no funding sources to disclose.

\section{Author Contributions}

All the authors contributed to the design, writing, and revision of the manuscript.
Volpe/Gallo/Zieroth 


\section{References}

1 McMurray JJV, Solomon SD, Inzucchi SE, Køber L, Kosiborod MN, Martinez FA, et al. Dapagliflozin in patients with heart failure and reduced ejection fraction. N Engl J Med. 2019 Nov 21;381(21):1995-2008.

2 Packer M, Anker SD, Butler J, Filippatos G, Pocock SJ, Carson P, et al. Cardiovascular and renal outcomes with empagliflozin in heart failure. N Engl J Med. 2020 Oct 8;383(15): 1413-24.

3 McDonagh TA, Metra M, Adamo M, Gardner RS, Baumbach A, Böhm M, et al. 2021 ESC Guidelines for the diagnosis and treatment of acute and chronic heart failure. Eur Heart J. 2021 Aug 27;42(36):3599.

4 Volpe M, Bauersachs J, Bayés-Genís A, Butler J, Cohen-Solal A, Gallo G, et al. Sacubitril/valsartan for the management of heart failure: a perspective viewpoint on current evidence. Int J Cardiol. 2021 Mar 15;327:138-45.

5 Packer M, Anker SD, Butler J, Filippatos G, Ferreira JP, Pocock SJ, et al. Influence of neprilysin inhibition on the efficacy and safety of empagliflozin in patients with chronic heart failure and a reduced ejection fraction: the EMPEROR-Reduced trial. Eur Heart J. 2021 Feb 11;42(6):671-80.

6 Solomon SD, Jhund PS, Claggett BL, Dewan P, Køber L, Kosiborod MN, et al. Effect of Dapagliflozin in patients with HFrEF treated with sacubitril/valsartan: the DAPA-HF trial. JACC Heart Fail. 2020 Oct;8(10):811-8.
7 Docherty KF, Jhund PS, Inzucchi SE, Køber L, Kosiborod MN, Martinez FA, et al. Effects of dapagliflozin in DAPA-HF according to background heart failure therapy. Eur Heart J. 2020 Jul 1;41(25):2379-92.

8 Bhatt DL, Szarek M, Steg PG, Cannon CP, Leiter LA, McGuire DK, et al. Sotagliflozin in patients with diabetes and recent worsening heart failure. N Engl J Med. 2021 Jan 14; 384(2):117-28.

9 Zannad F, Ferreira JP, Pocock SJ, Anker SD, Butler J, Filippatos G, et al. SGLT2 inhibitors in patients with heart failure with reduced ejection fraction: a meta-analysis of the EMPEROR-Reduced and DAPA-HF trials. Lancet. 2020 Sep 19;396(10254):819-29.

10 Hsiao FC, Lin CP, Tung YC, Chang PC, McMurray JJV, Chu PH. Combining sodiumglucose cotransporter 2 inhibitors and angiotensin receptor-neprilysin inhibitors in heart failure patients with reduced ejection fraction and diabetes mellitus: a multi-institutional study. Int J Cardiol. 2021 May 1;330:91-7.

11 Jiménez-Blanco Bravo M, Valle A, Ordás JG, Díaz SDP, Pereda DC, Climent HM, et al. Safety and efficacy of the combination of sacubitril/valsartan and SGLT2i in HFrEF patients (SECSI Registry). J Cardiovasc Pharmacol. 2021 Jul 19.

12 Ahmad T, Desai NR. Quadruple therapy is the new standard of care for HFrEF. JACC Heart Fail. 2020 Oct;8(10):819-21.

13 Bauersachs J. Heart failure drug treatment: the fantastic four. Eur Heart J. 2021 Feb 11; 42(6):681-3.
14 McMurray JJV, Packer M. How should we sequence the treatments for heart failure and a reduced ejection fraction? A redefinition of evidence-based medicine. Circulation. 2021 Mar 2;143(9):875-7.

15 McMurray JJ, Packer M, Desai AS, Gong J, Lefkowitz MP, Rizkala AR, et al. Angiotensinneprilysin inhibition versus enalapril in heart failure. N Engl J Med. 2014 Sep 11;371(11): 993-1004.

16 Januzzi JL Jr, Prescott MF, Butler J, Felker GM, Maisel AS, McCague K, et al. Association of change in $\mathrm{N}$-terminal pro-B-type natriuretic peptide following initiation of sacubitril-valsartan treatment with cardiac structure and function in patients with heart failure with reduced ejection fraction. JAMA. 2019 Sep 2;322(11):1-11.

17 Wachter R, Senni M, Belohlavek J, Straburzynska-Migaj E, Witte KK, Kobalava Z, et al. Initiation of sacubitril/valsartan in haemodynamically stabilised heart failure patients in hospital or early after discharge: primary results of the randomised TRANSITION study. Eur J Heart Fail. 2019 Aug;21(8):998-1007.

18 Senni M, McMurray JJ, Wachter R, McIntyre HF, Reyes A, Majercak I, et al. Initiating sacubitril/valsartan (LCZ696) in heart failure: results of TITRATION, a double-blind, randomized comparison of two uptitration regimens. Eur J Heart Fail. 2016 Sep;18(9): 1193-202. 